\title{
Differential Geometry of the Lie algebra of the quantum plane
}

\author{
Salih Çelik ${ }^{1}$ and Sultan A Çelik \\ Yildiz Technical University, Department of Mathematics, \\ 34210 Davutpasa-Esenler, Istanbul, TURKEY.
}

\begin{abstract}
We present a differential calculus on the extension of the quantum plane obtained considering that the (bosonic) generator $x$ is invertible and furthermore working polynomials in $\ln x$ instead of polynomials in $x$. We call quantum Lie algebra to this extension and we obtain its Hopf algebra structure and its dual Hopf algebra.
\end{abstract}

\footnotetext{
${ }^{1}$ E-mail address: sacelik@yildiz.edu.tr
} 
Quantum plane [1] is a simple example of quantum space and has been studied intensively by many authors in the past years. It can be obtained by deformation of the classical plane [2]. For references to the literature we refer to the recent book by Majid [3].

As usual in noncommutative geometry [4] quantum plane has many differential calculi. By using the noncommutative differential geometry method in Ref. 5 and interpreting the dual plane of the quantum plane to consist of differentials of the coordinates of the quantum plane, covariant differential calculus on the quantum plane has been developed. Therefore the quantum plane provides a simple example for noncommutative differential geometry [6].

It is known that, in order to construct a noncommutative differential calculus on quantum groups and Hopf algebras, one takes into consideration the associative algebra of functions on the group. The starting point of this work is its Lie algebra. For quamtum superplane, similar a work studied in Ref. 7. In this work, we present here a differential calculus on the Lie algebra of the associative algebra of functions on the extended quantum plane.

The quantum plane [1] is defined as an associative algebra generated by two noncommuting coordinates $x$ and $y$ with the single quadratic relation

$$
x y-q y x=0 \quad q \in \mathcal{C}-\{0\} .
$$

This algebra is known as the algebra of polynomials over the quantum plane and we shall denote by $\mathcal{A}_{q}=\operatorname{Fun}_{q}(R(2))$. We extend the algebra $\mathcal{A}_{q}$ by including inverse of $x$ which obeys

$$
x x^{-1}=1=x^{-1} x .
$$

We know, from Ref. 8, that the extended algebra $\mathcal{A}_{q}$ is a Hopf algebra with the following costructures:

$$
\begin{gathered}
\Delta(x)=x \otimes x \quad \Delta(y)=y \otimes 1+x \otimes y \\
\epsilon(x)=1 \quad \epsilon(y)=0 \\
\kappa(x)=x^{-1} \quad \kappa(y)=-x^{-1} y .
\end{gathered}
$$

It is not difficult to verify the following properties of costructures:

$$
(\Delta \otimes \mathrm{id}) \circ \Delta=(\mathrm{id} \otimes \Delta) \circ \Delta
$$




$$
\begin{gathered}
m \circ(\epsilon \otimes \mathrm{id}) \circ \Delta=m \circ(\mathrm{id} \otimes \epsilon) \circ \Delta \\
m \circ(\kappa \otimes \mathrm{id}) \circ \Delta=\epsilon=m \circ(\mathrm{id} \otimes \kappa) \circ \Delta
\end{gathered}
$$

where id denotes the identity mapping and $m$ is the multiplication map

$$
m: \mathcal{A}_{q} \otimes \mathcal{A}_{q} \longrightarrow \mathcal{A}_{q} \quad m(a \otimes b)=a b
$$

It is known that an element of a Lie group can be represented by exponential of an element of its Lie algebra. Using this fact, we can define the generators of $\mathcal{A}_{q}$ as $[9]$

$$
x=e^{X} \quad y=e^{X} Y \quad q=e^{h} .
$$

Then we obtain the relation

$$
[X, Y]=h Y
$$

where

$$
[a, b]_{ \pm}=a b \pm b a
$$

This is the relations of a Lie algebra and we shall denote it by $\mathcal{L}\left(\mathcal{A}_{q}\right)=: g_{h}$. The Hopf algebra structure of $g_{h}$ can be read off from (2),

$$
\begin{gathered}
\Delta(X)=X \otimes 1+1 \otimes X \\
\Delta(Y)=Y \otimes e^{-X}+1 \otimes Y \\
\epsilon(X)=0 \quad \epsilon(Y)=0 \\
\kappa(X)=-X \quad \kappa(Y)=-Y e^{X} .
\end{gathered}
$$

We now shall build up a noncommutative differential calculus on the Lie algebra $g_{h}$. This must be involve functions on the Lie algebra $g_{h}$, differentials and differential forms. For this, we have to define a linear map $\delta$ which acts on the functions of the elements of $g_{h}$. The exterior differential $\delta$ is an operator which gives the mapping from the generators of $g_{h}$ to the differentials:

$$
\delta: u \mapsto \delta u \quad u \in\{X, Y\}
$$

We demand that the exterior differential $\delta$ has to satisfy two properties: the nilpotency

$$
\delta^{2}=0
$$


and the Leibniz rule

$$
\delta(f g)=(\delta f) g+f(\delta g) .
$$

In order to establish a noncommutative differential calculus on the algebra $g_{h}$, we assume that the commutation relations between the elements of $g_{h}$ and their differentials are the following form:

$$
\begin{aligned}
& X \delta X=A_{11} \delta X X+B_{1} \delta X+B_{2} \delta Y \\
& X \delta Y=A_{12} \delta Y X+B_{3} \delta X+B_{4} \delta Y \\
& Y \delta X=A_{21} \delta X Y+B_{5} \delta X+B_{6} \delta Y \\
& Y \delta Y=A_{22} \delta Y Y+B_{7} \delta X+B_{8} \delta Y .
\end{aligned}
$$

The coefficients $A_{i j}$ and $B_{i}$ will be determined in terms of the "new" deformation parameter $h$. To find them we shall use the consistency of calculus.

Using the consistency conditions

$$
\begin{gathered}
\delta(X Y-Y X-h Y)=0 \\
(X Y-Y X-h Y) \delta X=0 \\
(X Y-Y X-h Y) \delta Y=0
\end{gathered}
$$

we obtain the system

$$
\begin{gathered}
A_{11}=A_{12}=A_{21}=0 \quad B_{2}\left(1-A_{22}\right)=0 \\
B_{3}-B_{5}=0 \quad B_{4}-B_{6}=h \quad B_{3} B_{6}-B_{2} B_{7}=h B_{5} \\
\left(B_{1}-B_{4}\right) B_{6}+\left(B_{8}-B_{5}\right) B_{2}=-h B_{6} \\
B_{3} B_{6}-B_{2} B_{7}=h B_{8} \\
\left(B_{1}-B_{4}\right) B_{7}+\left(B_{8}-B_{5}\right) B_{3}=h B_{7}
\end{gathered}
$$

or

$$
\begin{gathered}
A_{11}=A_{12}=A_{21}=0 \quad B_{2}\left(1-A_{22}\right)=0 \\
B_{3}=B_{5}=B_{8} \quad B_{4}-B_{6}=h \quad\left(B_{1}-B_{4}-h\right) B_{7}=0 .
\end{gathered}
$$

Thus we have the following algebra

$$
[X, \delta X]=B_{1} \delta X+B_{2} \delta Y
$$




$$
\begin{gathered}
{[X, \delta Y]=B_{3} \delta X+B_{4} \delta Y} \\
{[Y, \delta X]=B_{3} \delta X+\left(B_{4}-h\right) \delta Y} \\
{[Y, \delta Y]=\left(A_{22}-1\right) \delta Y Y+B_{7} \delta X+B_{3} \delta Y .}
\end{gathered}
$$

But we do not have the required relations because of the constants. To find the constans in the above relations, we shall consider the covariance of the noncommutative differential calculus.

We first note that consistency of a differential calculus with commutation relation (5) means that the algebra is a graded associative algebra generated by the elements of the set $\{X, Y, \delta X, \delta Y\}$. So, it is sufficient only describe the actions of co-maps on the subset $\{\delta X, \delta Y\}$.

Let $\Gamma$ be a free left module over the algebra $g_{h}$ generated by the elements of the set $\{X, Y, \delta X, \delta Y\}$. The module $\Gamma$ becomes a unital associative algebra if one defines a multiplication law on $\Gamma$ by relations (5) and (9).

We consider a map $\phi_{L}: \Gamma \longrightarrow g_{h} \otimes \Gamma$ such that

$$
\phi_{L} \circ \delta=(\mathrm{id} \otimes \delta) \circ \Delta
$$

Thus we have

$$
\phi_{L}(\delta X)=1 \otimes \delta X \quad \phi_{L}(\delta Y)=1 \otimes \delta Y+\frac{1-e^{h}}{h} Y \otimes X e^{-X} .
$$

We now define a map $\Delta_{L}$ as follows:

$$
\Delta_{L}\left(a_{1} \delta b_{1}+\delta b_{2} a_{2}\right)=\Delta\left(a_{1}\right) \phi_{L}\left(\delta b_{1}\right)+\phi_{L}\left(\delta b_{2}\right) \Delta\left(a_{2}\right)
$$

Applying the linear map $\Delta_{L}$ to relations (9) we get, as the solution of the obtained system,

$$
\begin{gathered}
A_{11}=A_{12}=A_{21}=1 \quad A_{22}=e^{h} \\
B_{1}=B_{6}=-h \quad B_{2}=B_{3}=B_{4}=B_{5}=B_{7}=B_{8}=0 .
\end{gathered}
$$

Consequently, the relations (9) is of the form

$$
\begin{gathered}
{[X, \delta X]=-h \delta X \quad[X, \delta Y]=0} \\
{[Y, \delta X]=-h \delta Y \quad[Y, \delta Y]=\left(e^{h}-1\right) \delta Y Y .}
\end{gathered}
$$


Applying the exterior differential $\delta$ to the relation (14) one has

$$
[\delta X, \delta Y]_{+}=0 \quad(\delta X)^{2}=0=(\delta Y)^{2} .
$$

Note that, the differentials of $X$ and $Y$ in terms of $x$ and $y$ as follows:

$$
\delta X=\frac{h}{1-e^{-h}} \delta x x^{-1} \quad \delta Y=x^{-1}\left(\delta y-\delta x x^{-1} y\right) .
$$

We now consider another map $\phi_{R}: \Gamma \longrightarrow \Gamma \otimes g_{h}$ such that

$$
\phi_{R} \circ \delta=(\delta \otimes \mathrm{id}) \circ \Delta .
$$

Thus one has

$$
\phi_{R}(\delta X)=\delta X \otimes 1 \quad \phi_{R}(\delta Y)=\delta Y \otimes e^{-X} .
$$

We define a map $\Delta_{R}$ with again (12) by replacing $R$ with $L$. The map $\Delta_{R}$ also leaves invariant the relations (14) and (15), and the following identities are satisfied:

$$
\begin{array}{ll}
(\Delta \otimes \mathrm{id}) \circ \Delta_{L}=\left(\mathrm{id} \otimes \Delta_{L}\right) \circ \Delta_{L} & (\epsilon \otimes \mathrm{id}) \circ \Delta_{L}=\text { id } \\
(\mathrm{id} \otimes \Delta) \circ \Delta_{R}=\left(\Delta_{R} \otimes \mathrm{id}\right) \circ \Delta_{R} & (\text { id } \otimes \epsilon) \circ \Delta_{R}=\text { id } .
\end{array}
$$

To denote the coproduct, counit and coinverse which will be defined on the algebra $\Gamma$ with those of $\mathcal{A}$ may be inadvisable. For this reason, we shall denote them with a different notation. Let us define the map $\hat{\Delta}$ as

$$
\hat{\Delta}=\Delta_{R}+\Delta_{L}
$$

which will allow us to define the coproduct of the differential algebra. We denote the restriction of $\hat{\Delta}$ to the algebra $g_{h}$ by $\Delta$ and the extension of $\Delta$ to the differential algebra $\Gamma$ by $\hat{\Delta}$. It is possible to interpret the relation

$$
\left.\hat{\Delta}\right|_{g_{h}}=\Delta
$$

as the definition of $\hat{\Delta}$ on the generators of $g_{h}$ and (19) as the definition of $\hat{\Delta}$ on differentials.

It is not difficult to verify the following conditions: 
a) $\Gamma$ is an $g_{h}$-bimodule,

b) $\Gamma$ is an $g_{h}$-bicomodule with left and right coactions $\Delta_{R}$ and $\Delta_{L}$, respectively, making $\Gamma$ a left and right $g_{h}$-comodule with (18), and

$$
\left(\Delta_{L} \otimes \mathrm{id}\right) \circ \Delta_{R}=\left(\mathrm{id} \otimes \Delta_{R}\right) \circ \Delta_{L}
$$

which is the $g_{h}$-bimodule property. So, the triple $\left(\Gamma, \Delta_{L}, \Delta_{R}\right)$ is a bicovariant bimodule over Hopf algebra $g_{h}$. In additional, since

c) $(\Gamma, \delta)$ is a first order differential calculus over $g_{h}$, and

d) $\delta$ is both a left and a right comodule map, i.e. for all $a \in g_{h}$

$$
(\mathrm{id} \otimes \delta) \Delta(a)=\Delta_{L}(\delta a) \quad(\delta \otimes \mathrm{id}) \Delta(a)=\Delta_{R}(\delta a)
$$

the quadruple $\left(\Gamma, d, \Delta_{L}, \Delta_{R}\right)$ is a first order bicovariant differential calculus over Hopf algebra $g_{h}$.

Now let us return Hopf algebra structure of $\Gamma$. If we define a counit $\hat{\epsilon}$ for the differential algebra as

$$
\hat{\epsilon} \circ \delta=\delta \circ \epsilon=0
$$

and

$$
\left.\hat{\epsilon}\right|_{g_{h}}=\left.\epsilon \quad \epsilon\right|_{\Gamma}=\hat{\epsilon}
$$

we have

$$
\hat{\epsilon}(\delta X)=0 \quad \hat{\epsilon}(\delta Y)=0
$$

where

$$
\hat{\epsilon}\left(a_{1} \delta b_{1}+\delta b_{2} a_{2}\right)=\epsilon\left(a_{1}\right) \hat{\epsilon}\left(\delta b_{1}\right)+\hat{\epsilon}\left(\delta b_{2}\right) \epsilon\left(a_{2}\right) .
$$

Here we used the fact that $\delta(1)=0$.

As the next step we obtain a coinverse $\hat{\kappa}$. For this, it suffices to define $\hat{\kappa}$ such that

$$
\hat{\kappa} \circ \delta=\delta \circ \kappa
$$

and

$$
\left.\hat{\kappa}\right|_{g_{h}}=\left.\kappa \quad \kappa\right|_{\Gamma}=\hat{\kappa}
$$

where

$$
\hat{\kappa}\left(a_{1} \delta b_{1}+\delta b_{2} a_{2}\right)=\hat{\kappa}\left(\delta b_{1}\right) \kappa\left(a_{1}\right)+\kappa\left(a_{2}\right) \hat{\kappa}\left(\delta b_{2}\right) .
$$


Thus the action of $\hat{\kappa}$ on the generators $\delta X$ and $\delta Y$ is as follows:

$$
\hat{\kappa}(\delta X)=-\delta X \quad \hat{\kappa}(\delta Y)=e^{X-h}\left(\delta Y+\frac{e^{h}-1}{h} \delta X Y\right)
$$

Note that it is easy to check that $\hat{\epsilon}$ and $\hat{\kappa}$ leave invariant the relations (14) and (15). Consequently, we can say that the structure $(\Gamma, \hat{\Delta}, \hat{\epsilon}, \hat{\kappa})$ is a graded Hopf algebra.

Just as we introduced the derivatives of the generators of $\mathcal{A}_{q}$ in the standard way, let us introduce derivatives of the generators of $g_{h}$ and multiply explicit expression of the exterior differential $\delta$ from the right by $X f$ and $Y f$, respectively. Then, using the Leibniz rule for partial derivatives

$$
\partial_{i}(f g)=\left(\partial_{i} f\right) g+f\left(\partial_{i} g\right)
$$

we obtain

$$
\begin{gathered}
{\left[\partial_{X}, X\right]=\frac{h}{1-e^{-h}}\left(1+\left(e^{-h}-1\right) \partial_{X}\right)} \\
{\left[\partial_{X}, Y\right]=0 \quad\left[\partial_{Y}, X\right]=0} \\
{\left[\partial_{Y}, Y\right]=e^{h}+\left(1-e^{h}\right)\left(\partial_{X}-Y \partial_{Y}\right) .}
\end{gathered}
$$

Note that, the partial derivatives of $X$ and $Y$ in terms of $x$ and $y$ as follows:

$$
\partial_{X}=x \partial_{x}+y \partial_{y} \quad \partial_{Y}=e^{h} x \partial_{y}
$$

The commutation relations among the partial derivatives can be easily obtained by using $\delta^{2}=0$. So it follows that

$$
\begin{aligned}
\delta & =\frac{1-e^{-h}}{h} \delta X \partial_{X}+e^{-h} \delta Y \partial_{Y} \\
0 & =\delta^{2}=\delta\left(\frac{1-e^{-h}}{h} \delta X \partial_{X}+e^{-h} \delta Y \partial_{Y}\right) \\
& =e^{-h} \frac{e^{-h}-1}{h} \delta X \delta Y\left(\partial_{Y} \partial_{X}-\partial_{X} \partial_{Y}\right)
\end{aligned}
$$

which says that

$$
\left[\partial_{X}, \partial_{Y}\right]=0
$$


In order to find the commutation relations between the differentials and partial derivatives, we demand a form similar to (8). After some tedious but straigtword calculations, we find

$$
\begin{gathered}
{\left[\partial_{X}, \delta X\right]=\left(e^{h}-1\right) \delta X \partial_{X}-e^{h} \delta X} \\
{\left[\partial_{X}, \delta Y\right]=\left(e^{h}-1\right) \delta Y \partial_{X}-e^{h} \delta Y} \\
{\left[\partial_{Y}, \delta X\right]=0} \\
{\left[\partial_{Y}, \delta Y\right]=\left(e^{h}-1\right) \delta Y \partial_{Y}+\frac{\left(e^{h}-1\right)^{2}}{h} \delta X \partial_{X}+e^{h} \frac{1-e^{h}}{h} \delta X .}
\end{gathered}
$$

We now define two one-forms using the generators of $g_{h}$ and show that the algebra of forms is a graded Hopf algebra.

If we call them $w_{1}$ and $w_{2}$ then we can define them as follows:

$$
w_{1}=\frac{1-e^{-h}}{h} \delta X \quad w_{2}=e^{X} \delta Y .
$$

We denote the algebra of forms generated by $w_{1}$ and $w_{2}$ by $\Omega$. The generators of the algebra $\Omega$ with the generators of $g_{h}$ satisfy the following relations:

$$
\begin{gathered}
{\left[w_{1}, X\right]=h w_{1} \quad\left[w_{1}, Y\right]=\left(1-e^{-h}\right) e^{-X} w_{2}} \\
{\left[w_{2}, X\right]=0 \quad\left[w_{2}, Y\right]=0 .}
\end{gathered}
$$

The commutation rules of the generators of $\Omega$ are

$$
w_{1} w_{2}=-e^{h} w_{2} w_{1} \quad w_{1}^{2}=0=w_{2}^{2} .
$$

One can make the algebra $\Omega$ into a graded Hopf algebra with the following costructures: the coproduct $\Delta: \Omega \longrightarrow \Omega \otimes \Omega$ is given by

$$
\begin{gathered}
\Delta\left(w_{1}\right)=w_{1} \otimes 1+1 \otimes w_{1} \\
\Delta\left(w_{2}\right)=w_{2} \otimes 1+e^{X} \otimes w_{2}-e^{X} Y \otimes w_{1} .
\end{gathered}
$$

The counit $\epsilon: \Omega \longrightarrow \mathcal{C}$ is given by

$$
\epsilon\left(w_{1}\right)=0 \quad \epsilon\left(w_{2}\right)=0
$$


and the coinverse $\kappa: \Omega \longrightarrow \Omega$ is defined by

$$
\kappa\left(w_{1}\right)=-w_{1} \quad \kappa\left(w_{2}\right)=e^{-X} w_{2}+Y w_{1} .
$$

Now we shall obtain the universal enveloping algebra of $g_{h}, \mathcal{U}\left(g_{h}\right)$, via the vector fields related to the one-forms. We first write the Cartan-Maurer forms as

$$
\delta X=\frac{h}{1-e^{-h}} w_{1} \quad \delta Y=e^{-X} w_{2} .
$$

Writing the exterior differential $\delta$ in the form

$$
\delta=w_{1} T_{1}+w_{2} T_{2}
$$

and considering an arbitrary function $f$ of the generators of $g_{h}$ and using the nilpotency of $\delta$ one has

$$
T_{2} T_{1}=e^{-h} T_{1} T_{2}+T_{2} .
$$

The commutation relations of vector fields with the generators of $g_{h}$ as follows:

$$
\begin{gathered}
{\left[T_{1}, X\right]=\frac{h}{1-e^{-h}}\left(1+\left(e^{-h}-1\right) T_{1}\right)} \\
{\left[T_{1}, Y\right]=0 \quad\left[T_{2}, X\right]=0} \\
{\left[T_{2}, Y\right]=e^{-X}\left(1+\left(e^{-h}-1\right) T_{1}\right) .}
\end{gathered}
$$

The Hopf algebra structure of the vector fields is given by

$$
\begin{gathered}
\Delta\left(T_{1}\right)=T_{1} \otimes 1+1 \otimes T_{1}+\left(e^{-h}-1\right) T_{1} \otimes T_{1} \\
\Delta\left(T_{2}\right)=T_{2} \otimes 1+1 \otimes T_{2}+\left(e^{-h}-1\right) T_{2} \otimes T_{1} \\
\epsilon\left(T_{1}\right)=0 \quad \epsilon\left(T_{2}\right)=0 \\
\kappa\left(T_{1}\right)=-T_{1}\left(1+\left(e^{-h}-1\right) T_{1}\right)^{-1} \\
\kappa\left(T_{2}\right)=-T_{2}\left\{1-\left(e^{-h}-1\right) T_{1}\left(1+\left(e^{-h}-1\right) T_{1}\right)^{-1}\right\} .
\end{gathered}
$$

If we introduce the operators $H$ and $N$ as

$$
T_{1}=\frac{e^{-h N}-1}{e^{-h}-1} \quad \text { and } \quad T_{2}=e^{-h N} H
$$


we get

$$
[H, N]=H .
$$

Then, dual Hopf algebra to $\mathcal{U}\left(g_{h}\right)$ is given by

$$
\begin{gathered}
\Delta(N)=N \otimes 1+1 \otimes N \\
\Delta(H)=H \otimes 1+e^{h N} \otimes H \\
\epsilon(N)=0 \quad \epsilon(H)=0 \\
\kappa(N)=-N \quad \kappa(H)=-e^{-h N} H .
\end{gathered}
$$

\section{Acknowledgment}

This work was supported in part by TBTAK the Turkish Scientific and Technical Research Council.

\section{References}

[1] Manin Yu I 1988 (Montreal Univ Preprint)

[2] Fairlie D and Zachos C 1991 Phys Lett B 25643

[3] Majid S 1995 "Foundations of Quantum Group Theory" (Cambridge Univ Press)

[4] Connes A 1994 "Noncommutative Differential Geometry" (Academic Press San Diego)

[5] Woronowicz S L 1987 Commun Math Phys 111 613; 1989 ibid 122125

[6] Wess J and Zumino B 1990 Nucl Phys (Proc Suppl) 18 B 302

[7] Celik S 2002 q-alg/0201170

[8] Brzezinski T and Majid S 1992 Lett Math Phys 26 67;

Brzezinski T 1993 Lett Math Phys 27 287;

Celik S A and Celik S 2000 Int J Mod Phys A 153237

[9] Fronsdal C and Galindo A 1993 Lett Math Phys 2759 